\title{
Intervention Type
}

National Cancer Institute

\section{Source}

National Cancer Institute. Intervention Type. NCI Thesaurus. Code C98747.

The kind of product or procedure studied in a trial. 